\title{
Fusarium-Damaged Kernels and Deoxynivalenol in Fusarium-Infected U.S. Winter Wheat
}

\author{
Feng Jin, Guihua Bai, Dadong Zhang, Yanhong Dong, Lingjian Ma, William Bockus, and Floyd Dowell
}

First and fifth authors: College of Agronomy, Northwest A\&F University, Yangling, 712100, Shaanxi, China; first, second, and third authors: Department of Agronomy, Kansas State University, Manhattan 66506; second author: United States Department of AgricultureAgricultural Research Service (USDA-ARS), Hard Winter Wheat Genetics Research Unit, Manhattan, KS 66506; fourth author: Department of Plant Pathology, University of Minnesota, St. Paul 55108; sixth author: Department of Plant Pathology, Kansas State University, Manhattan; and seventh author: USDA-ARS Engineering and Wind Erosion Research Unit, Manhattan, KS 66502.

Accepted for publication 20 December 2013.

\section{ABSTRACT}

Jin, F., Bai, G.-H., Zhang, D.-D., Dong, Y.-H., Ma, L.-J., Bockus, W., and Dowell, F. 2014. Fusarium-damaged kernels and deoxynivalenol in Fusarium-infected U.S. winter wheat. Phytopathology 104:472-478.

Fusarium head blight (FHB) is a devastating disease that threatens wheat (Triticum aestivum) production in many areas worldwide. FHB infection results in Fusarium-damaged kernels (FDK) and deoxynivalenol (DON) that dramatically reduce grain yield and quality. More effective and accurate disease evaluation methods are imperative for successful identification of FHB-resistant sources and selection of resistant cultivars. To determine the relationships among different types of resistance, 363 (74 soft and 289 hard) U.S. winter wheat accessions were repeatedly evaluated for FDK and DON concentration in greenhouse and field experiments. Single-kernel near-infrared (SKNIR)-estimated FDK and DON were compared with visually estimated FDK and gas chromatographymass spectroscopy-estimated DON. Significant correlations were detected between percentage of symptomatic spikelets and visual FDK in the greenhouse and field, although correlations were slightly lower in the field. High correlation coefficients also were observed between visually scored FDK and SKNIR-estimated FDK $(0.72, P<0.001)$ and SKNIRestimated DON $(0.68, P<0.001)$; therefore, both visual scoring and SKNIR methods are useful for estimating FDK and DON in breeding programs.
Fusarium head blight (FHB) is a destructive disease of wheat worldwide. In the United States, Fusarium graminearum Schwabe (teleomorph $=$ Gibberella zeae (Schwein.) Petch) is the prevailing species responsible for FHB (4). FHB causes significant reductions in both grain yield and quality through Fusarium-damaged kernels (FDK) and mycotoxins, especially deoxynivalenol (DON), produced in harvested grain infected by the pathogen (4). DON contamination is a serious health concern to humans and livestock. Maximum allowable DON concentration in wheat grain for human consumption ranges from 0.5 to $2.0 \mathrm{mg} / \mathrm{kg}$ depending on the country $(4,23)$; therefore, in the regions where FHB epidemics are frequent and severe, FHB threatens wheat production.

Fusarium infection results in FDK: whitish, shrived kernels or tombstones that not only reduce kernel weight but also damage the protein quality. Wheat FDK are contaminated with DON in most FHB epidemic regions (4,14). Resistant cultivars usually have reduced DON concentrations (type III resistance) and fewer FDK (type IV resistance) than susceptible cultivars after infection (15). To date, these two types of resistance have not been systematically characterized in U.S. winter wheat. Some reports have suggested that FHB severity and FDK could predict DON

Corresponding authors: G.-H. Bai; E-mail address: guihua.bai@ars.usda.gov L.-J. Ma; E-mail address: malingjian@nwsuaf.edu.cn

* The $\boldsymbol{e}$-Xtra logo stands for "electronic extra" and indicates that the online version contains one supplemental table.

http://dx.doi.org/10.1094/PHYTO-07-13-0187-R

This article is in the public domain and not copyrightable. It may be freely reprinted with customary crediting of the source. The American Phytopathological Society, 2014. concentration but the conclusions on the relationships among these three measurements of FHB remain equivocal $(3,10,12$, 19,25).

Although a worldwide effort has been made to identify FHBresistant germplasm (4), only Sumai 3 and its derivatives have been identified as having a major quantitative trait locus (QTL) (Fhbl) for type II resistance (FHB spread within a spike); this QTL has the greatest and most stable effect on FHB resistance across different genetic backgrounds and environments among the FHB resistance sources identified to date $(1,2,5,13,24)$ and has been used extensively as a major source of resistance in breeding programs $(4,22)$. The level of the Fhbl contribution to lessen FDK and DON concentration in U.S. winter wheat remains unknown.

A number of technologies are available to quantify DON concentration in Fusarium-contaminated grain and each has its strength and weaknesses $(3,17,21)$. All these measuring methods are complicated; however, a simpler visual scoring method may be sufficient for breeders to quickly estimate DON concentration in breeding materials. This study was designed to identify an effective method for determining FDK and DON by evaluating the relationships among different measurements of FHB resistance. Meanwhile, this study also identified U.S. wheat germplasm with low DON concentration and FDK and assessed the effect of Fhbl on FDK and DON concentration in these germplasm lines.

\section{MATERIALS AND METHODS}

Plant materials and FHB inoculation. Two sets of wheat materials consisting of 363 wheat accessions were evaluated for FHB resistance in both the greenhouse and field experiments from 2009 through 2012 as described by Jin et al. (9). Set I had 
207 accessions, including all of the hard winter wheat (HWW) and soft winter wheat (SWW) entries from the 2008 nurseries and breeding lines from Oklahoma; and set II had 191 accessions, including 156 new accessions from the $2010 \mathrm{HWW}$ nurseries and 35 selected accessions from set I. Greenhouse experiments were conducted at Kansas State University. After vernalization at $5^{\circ} \mathrm{C}$ in a cold chamber for 8 weeks, six wheat seedlings per accession were transferred into a 13-by-13-cm Dura-pot (Hummert Int., Earth City, MO) and grown at $19 \pm 2{ }^{\circ} \mathrm{C}$ at night and $22 \pm 2^{\circ} \mathrm{C}$ during the day with a 12 -h photoperiod. Each experiment used a randomized complete block design with two replications. Six spikes per replication (pot) were inoculated at anthesis by injecting $10 \mu \mathrm{l}$ of conidia $(\approx 100$ spores/ $\mu \mathrm{l})$ with a syringe into a central spikelet of each spike. Inoculum was a field isolate (GZ 3639 ) of $F$. graminearum from Kansas (6), which was increased in a mung bean broth for inoculation (9). Inoculated plants were enclosed in a moist chamber for $48 \mathrm{~h}$ at $22 \pm 2{ }^{\circ} \mathrm{C}$ to initiate infection, then moved to a greenhouse bench for disease development under the same conditions.

Field experiments were conducted in the FHB Nursery of the Plant Pathology Department, Kansas State University at Rocky Ford in Manhattan. In all, $\approx 40$ seeds per accession were planted in a 1-m-long single-row plot, and each experiment used a randomized complete block design with two replications. Spawn inoculation was implemented twice by scattering $F$. graminearum-infested corn kernels on the soil surface before boot stage (Feekes 8) and 2 weeks after (Feekes 10.1). In addition, single-spikelet inoculation was used to inoculate six spikes per plot using the same inoculum and the same method as described for the greenhouse experiments. The FHB nursery was sprinkled for $3 \mathrm{~min}$ every hour from 1900 to $0600 \mathrm{~h}$ daily from flowering to early dough stages to improve initial infection using an overhead impact sprinkler system.

Evaluation of FDK. In greenhouse experiments, inoculated plants from each pot were bulk harvested and manually threshed to insure that all infected shriveled kernels were collected. To score FDK, samples with 1, 5, 20, 50, 75, 95, and 100\% FDK were prepared as checks. FDK in each sample (at least 100 kernels) were visually inspected by comparing the grain sample with the checks. Each sample was graded by two scientists and the mean from the two readings was used for statistical analysis. Only accessions in set II were evaluated for FDK and DON in the greenhouse experiments.

In the field experiments, all plants from each plot (replication) were harvested by hand and threshed using a single plant thresher (Almaco, Nevada, IA) by leaving the air blower slightly open to keep as many tombstones as possible, then hand-cleaned to remove leftover trash. FDK were visually scored based on all grain (at least 1,000 kernels) harvested in each plot using the same method as that used in the greenhouse experiments.

DON analysis. In the greenhouse experiments, DON concentration (milligrams per kilogram) was measured for set II of 191 accessions (9) using gas chromatography-mass spectrometry (GC-MS) $(7,17)$ at the University of Minnesota, St. Paul. All grains harvested from inoculated plants in each line were weighed and extracted by soaking and shaking in acetonitrile/water (84/16, $\mathrm{vol} / \mathrm{vol}$ ) at a ratio of solvent (milliliters)/seed (grams) $\geq 12$ for $24 \mathrm{~h}$. The extract was passed through a column packed with $\mathrm{C}_{18}$ and aluminum oxide, and $1.5 \mathrm{ml}$ of filtrate was evaporated to dryness under nitrogen at room temperature, and derivatized by 25 trimethylsilyl ether (TMS) reagent $($ TMSI/TMCS $=100 / 1$, vol/vol). DON was analyzed by a GC-MS (Shimadzu GCMSQP2010; Shimadzu Corporation, Kyoto, Japan) using selected ion monitoring mode. The fragment ion $(\mathrm{m} / \mathrm{z}$ value) of 235.10 was used as target ion and 259.10 and 422.10 ions were used as reference ions. Concentration of DON in each sample was calculated using a 10-point standard calibration curve of 0.025 to $15 \mathrm{ng} / \mathrm{ml}$ generated with each set of samples.
In the field experiments, DON was measured by GC-MS using randomly sampled grain collected from each plot. All samples in set I collected in 2010 and 2011 and set II in 2011 and 2012 field experiments were evaluated for DON. In brief, a 4-g ground sample was extracted with $16 \mathrm{ml}$ of acetonitrile/water (84/16, $\mathrm{vol} / \mathrm{vol})$ for $1 \mathrm{~h}$. Extract $(4 \mathrm{ml})$ was passed through a column packed with $\mathrm{C}_{18}$ and aluminum oxide, and $1 \mathrm{ml}$ of filtrate was evaporated to dryness under nitrogen at room temperature and derivatized by $100 \mu \mathrm{l}$ of TMS reagent (TMSI/TMCS $=100 / 1$, vol/vol). DON was quantified using GC-MS with quantification limit at $50 \mathrm{ng} / \mathrm{g}$.

Data analysis. Analysis of variance and correlation coefficient were calculated using the Statistical Analysis System 9.2 (SAS Institute, Inc., Cary, NC). Percentage of symptomatic spikelets (PSS) reported in a previous study (9) was used to analyze the relationship between disease severity and FDK or DON concentrations. The contribution of $F h b 1$ in four different genetic backgrounds (Clark, Trego, Wesley, and Harding) was analyzed by comparing the mean FHB ratings of the Fhbl-containing nearisogenic lines (NILs) with their parents.

Single-kernel near-infrared for FDK and DON prediction. A subset of samples (100 accessions) from set I grown in the field in 2010 and all 191 samples from set II grown in the field in 2011 were evaluated for FDK, and 27 random samples from set I and 121 random samples from set II were evaluated for DON concentration using the single-kernel near-infrared (SKNIR) method. For the SKNIR method, 200 kernels/sample were separated into sound (bin 1) and FDK (bins 2 and 3 ) and the DON concentration was estimated in each kernel (21). The SKNIR system calibration for both FDK and DON followed Peiris et al. (21). Correlation coefficients were calculated among SKNIR, visually scored FDK, and SKNIR- and GC-MS-estimated DON concentrations.

\section{RESULTS}

FDK in the greenhouse and field experiments. In the greenhouse experiments, FDK varied significantly among 191 HWW accessions tested after Fusarium spp. infection, and $\approx 75 \%$ of accessions had mean FDK $>50 \%$, indicating that most U.S. HWW was susceptible to FHB infection. Only $10 \%$ of accessions tested had FDK $<25 \%$, and $15 \%$ had FDK of 25 to $50 \%$. Among the $10 \%$ low-FDK accessions, 5\%, including eight Fhbl NILs and KS08FHB-78 (Table 1), carry the Fhbl resistance allele, as determined by the closely linked markers (9).

Average FDK from the field experiments was much less than that from the greenhouse, and $\approx 73.6 \%$ of total tested accessions $(n=363)$ had FDK $<50.0 \%, 33.1 \%$ showed FDK $<25.0 \%$, and only $15.4 \%$ had $\leq 15.0 \%$ FDK in the field experiments. In all, $\approx 12.5 \%$ of the $15.4 \%$ low-FDK accessions carry the Fhbl resistance allele from the backcrossing project and had FDK $<25 \%$ in greenhouse experiments; meanwhile, $12.5 \%$ of other accessions without the Fhb1 resistance allele (T154, SD05085-1, Everest, Lyman, SD05118, Husker, and Overland) also showed a similar low FDK in the greenhouse experiments (Table 1). Among the remaining 75\% accessions, 23.2\% had FDK of 25 to $50 \%$ in the greenhouses, including four $F h b 1$ resistance allele carriers and nine non-Fhbl accessions such as Aspen, Heyne, Arapahoe, and so on; $51.8 \%$ other accessions had $>50 \%$ FDK $(51.7$ to $98 \%)$ in the greenhouses (Table 1$)$, indicating that the accessions with low FDK $(\leq 15 \%)$ in the field experiments might have relatively high FDK in the greenhouse experiments.

DON concentration of FHB-inoculated wheat in both the greenhouse and field. In the greenhouse experiments, all the samples from set II were evaluated for DON concentration using GC-MS. DON concentration was 0.42 to $1,003.4 \mathrm{mg} / \mathrm{kg}$, and only $1.0 \%$ of tested accessions had DON $<2.0 \mathrm{mg} / \mathrm{kg}$. DON concentration was arbitrarily classified into five groups $(<2.0,2.01$ to $5.0,5.01$ to $50.0,50.01$ to 100.0 , and $>100.0 \mathrm{mg} / \mathrm{kg}$ ); only $3.1 \%$ had DON concentration $<5.0 \mathrm{mg} / \mathrm{kg}$ and the majority $(96.9 \%)$ of 
accessions had $>5.0 \mathrm{mg} / \mathrm{kg} \mathrm{DON}$, including $22 \%$ the tested accessions that had DON concentrations of 5.01 to $50.0 \mathrm{mg} / \mathrm{kg}$, $19.4 \%$ that had $>50.0 \mathrm{mg} / \mathrm{kg}$ but $<100.0 \mathrm{mg} / \mathrm{kg}$, and the remaining $55.5 \%$ that had DON concentrations $>100 \mathrm{mg} / \mathrm{kg}$. In the $3.1 \%$ accessions with low DON concentration $(<5.0 \mathrm{mg} / \mathrm{kg})$, two-thirds carried Fhbl, including three WesleyFhb1 progenies and KS08FHB-78, and the remaining one-third (T154 and Everest) did not carry Fhbl (Table 1).

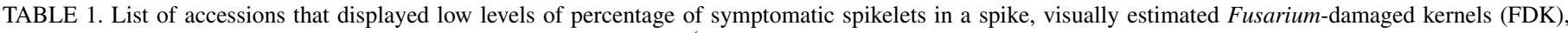
and gas chromatography-mass spectroscopy-measured deoxynivalenol (DON) content evaluated in greenhouse (GH) and field experiments ${ }^{\mathrm{a}}$

\begin{tabular}{|c|c|c|c|c|c|}
\hline Accession name & Type $^{\text {b }}$ & GH FDK $(\%)$ & GH DON (mg/kg) & Field FDK (\%) & Field DON (mg/kg) \\
\hline \multicolumn{6}{|l|}{ GH FDK $\leq 25 \%$} \\
\hline KS08FHB-78 & HWW & $5.3 \pm 3.7$ & $4.08 \pm 3.01$ & $23.8 \pm 15.6$ & $3.60 \pm 2.37$ \\
\hline WesleyFhb1NIL09S-103c & HWW & $5.3 \pm 3.7$ & $0.42 \pm 0.27$ & $9.0 \pm 8.7$ & $2.53 \pm 1.15$ \\
\hline WesleyFhb1NIL09S-104 ${ }^{c}$ & HWW & $8.7 \pm 5.8$ & $2.19 \pm 2.12$ & $23.8 \pm 21.6$ & $4.23 \pm 2.71$ \\
\hline WesleyFhb1NIL09S-105c & HWW & $10.0 \pm 7.1$ & $2.22 \pm 1.40$ & $4.1 \pm 3.8$ & $2.30 \pm 0.92$ \\
\hline TregoFhb1NIL09S-98 & HWW & $10.3 \pm 7.8$ & $5.15 \pm 5.44$ & $11.3 \pm 10.8$ & $6.46 \pm 1.70$ \\
\hline Wesley FHB1 & HWW & $15.0 \pm 4.1$ & $6.93 \pm 3.95$ & $6.5 \pm 6.2$ & $2.70 \pm 1.60$ \\
\hline TregoFhb1NIL09S-99 & HWW & $15.0 \pm 4.1$ & $9.57 \pm 11.86$ & $5.5 \pm 2.9$ & $4.07 \pm 1.41$ \\
\hline ClarkFhb1NIL09F-23 ${ }^{\mathrm{d}}$ & SWW & $23.3 \pm 4.7$ & $24.22 \pm 24.63$ & $6.5 \pm 3.8$ & $0.88 \pm 0.35$ \\
\hline ClarkFhb1NIL-75 & SWW & $23.3 \pm 4.7$ & $14.55 \pm 8.26$ & $13.3 \pm 21.2$ & $0.49 \pm 0.28$ \\
\hline $\mathrm{T} 154^{\mathrm{c}}$ & HWW & $5.3 \pm 3.7$ & $1.68 \pm 0.54$ & $13.8 \pm 6.5$ & $3.36 \pm 1.77$ \\
\hline SD05085-1 & HWW & $12.3 \pm 9.5$ & $5.07 \pm 3.0$ & $14.3 \pm 10.2$ & $4.97 \pm 1.08$ \\
\hline Everest $^{\mathrm{c}}$ & HWW & $13.3 \pm 6.2$ & $3.26 \pm 2.22$ & $8.3 \pm 4.1$ & $2.30 \pm 0.70$ \\
\hline Lyman & HWW & $17.0 \pm 12.0$ & $8.24 \pm 10.04$ & $4.3 \pm 3.7$ & $2.57 \pm 0.37$ \\
\hline Harry & HWW & $20.0 \pm 14.1$ & $19.43 \pm 24.37$ & $52.5 \pm 20.5$ & $11.65 \pm 2.28$ \\
\hline SD05118 & HWW & $20.0 \pm 8.2$ & $20.13 \pm 11.78$ & $14.0 \pm 9.3$ & $6.43 \pm 1.45$ \\
\hline Husker & HWW & $20.3 \pm 15.9$ & $15.03 \pm 17.58$ & $13.8 \pm 7.4$ & $7.14 \pm 2.53$ \\
\hline $\mathrm{T} 153$ & HWW & $23.3 \pm 12.5$ & $6.26 \pm 3.39$ & $17.5 \pm 5.6$ & $4.10 \pm 1.92$ \\
\hline Overland & HWW & $25.0 \pm 4.1$ & $7.42 \pm 0.42$ & $15.0 \pm 10.8$ & $4.48 \pm 1.86$ \\
\hline SD08198 & HWW & $25.0 \pm 17.8$ & $9.93 \pm 3.27$ & $15.9 \pm 15.8$ & $6.82 \pm 3.64$ \\
\hline \multicolumn{6}{|c|}{ Field FDK $\leq 15 \%$ or $\mathrm{DON}<2 \mathrm{mg} / \mathrm{kg}$} \\
\hline ClarkFhb1NIL09F-45 & SWW & $26.7 \pm 20.1$ & $26.20 \pm 34.86$ & $3.0 \pm 2.0$ & $0.59 \pm 0.18$ \\
\hline KS08FHB-31 & HWW & $28.3 \pm 15.5$ & $42.76 \pm 39.41$ & $3.4 \pm 1.8$ & $2.56 \pm 1.23$ \\
\hline TregoFhb1NIL09S-100d & HWW & $33.3 \pm 4.7$ & $8.58 \pm 7.38$ & $4.1 \pm 3.8$ & $1.82 \pm 0.63$ \\
\hline ClarkFhb1NIL09F-4 ${ }^{\mathrm{d}}$ & SWW & $60.0 \pm 14.1$ & $33.69 \pm 15.61$ & $5.0 \pm 0.0$ & $1.16 \pm 0.52$ \\
\hline HardingFhb1NIL09S-108 & HWW & $70.0 \pm 14.1$ & $99.04 \pm 38.46$ & $6.9 \pm 5.9$ & $4.87 \pm 3.26$ \\
\hline HardingFhb1NIL09S-109 & HWW & $30.0 \pm 16.3$ & $11.28 \pm 3.38$ & $10.0 \pm 6.1$ & $5.48 \pm 2.98$ \\
\hline NE08527 & HWW & $43.3 \pm 20.5$ & $31.07 \pm 32.04$ & $4.3 \pm 3.7$ & $2.81 \pm 1.52$ \\
\hline Jerry & HWW & $43.3 \pm 20.9$ & $32.92 \pm 22.48$ & $6.8 \pm 6.0$ & $3.26 \pm 2.23$ \\
\hline KS08IFAFS1 & HWW & $98.0 \pm 2.2$ & $343.95 \pm 80.29$ & $7.6 \pm 5.4$ & $2.51 \pm 1.52$ \\
\hline Kharkof & HWW & $65.0 \pm 21.2$ & $38.76 \pm 15.36$ & $7.8 \pm 8.1$ & $2.48 \pm 1.96$ \\
\hline MTS0713 & HWW & $51.7 \pm 31.7$ & $172.46 \pm 230.58$ & $7.8 \pm 8.0$ & $3.34 \pm 1.92$ \\
\hline Heyne & HWW & $31.7 \pm 14.3$ & $19.22 \pm 20.65$ & $9.0 \pm 7.1$ & $5.67 \pm 1.48$ \\
\hline CA9W08-856 & HWW & $56.7 \pm 20.5$ & $77.84 \pm 67.21$ & $9.0 \pm 12.2$ & $3.09 \pm 1.42$ \\
\hline CA9W07-817 & HWW & $86.7 \pm 10.3$ & $259.04 \pm 44.77$ & $9.0 \pm 12.2$ & $3.23 \pm 2.57$ \\
\hline Bess & SWW & - & - & $9.2 \pm 5.3$ & $5.23 \pm 1.67$ \\
\hline Aspen & HWW & $26.7 \pm 12.5$ & $11.20 \pm 2.32$ & $9.5 \pm 3.6$ & $2.91 \pm 2.06$ \\
\hline B030543 & SWW & - & - & $10.0 \pm 4.1$ & $3.72 \pm 1.22$ \\
\hline Karl 92 & HWW & $80.0 \pm 16.3$ & $236.20 \pm 191.66$ & $10.3 \pm 9.1$ & $4.03 \pm 1.40$ \\
\hline SD07126 & HWW & $67.5 \pm 27.5$ & $63.10 \pm 44.40$ & $10.4 \pm 9.6$ & $4.53 \pm 3.62$ \\
\hline SD05W030 & HWW & $44.3 \pm 38.0$ & $91.22 \pm 126.62$ & $10.8 \pm 9.3$ & $2.93 \pm 1.58$ \\
\hline SD07220 & HWW & $51.7 \pm 34.2$ & $24.01 \pm 25.11$ & $11.3 \pm 5.4$ & $4.48 \pm 0.95$ \\
\hline U07-698-9 & HWW & $43.3 \pm 9.4$ & $15.61 \pm 9.49$ & $11.3 \pm 5.4$ & $6.66 \pm 2.37$ \\
\hline Camelot & HWW & $36.7 \pm 20.9$ & $53.53 \pm 58.82$ & $11.4 \pm 8.8$ & $5.59 \pm 4.04$ \\
\hline HV9W04-1594R & HWW & $88.3 \pm 9.4$ & $161.93 \pm 79.85$ & $11.5 \pm 9.3$ & $5.27 \pm 2.45$ \\
\hline IL02-18228d & SWW & - & - & $11.7 \pm 8.5$ & $1.88 \pm 1.22$ \\
\hline KS08P1-108 & HWW & $98.0 \pm 2.2$ & $265.43 \pm 50.36$ & $11.8 \pm 8.3$ & $3.99 \pm 1.28$ \\
\hline KY96C-0769-7-3 & SWW & - & - & $12.5 \pm 6.3$ & $4.88 \pm 2.32$ \\
\hline MD01W233-06-1 ${ }^{\mathrm{d}}$ & SWW & - & - & $12.5 \pm 6.3$ & $1.79 \pm 0.53$ \\
\hline HV9W05-1125R & HWW & $91.3 \pm 8.2$ & $316.09 \pm 153.39$ & $12.5 \pm 9.0$ & $5.37 \pm 2.83$ \\
\hline NE05548 & HWW & $87.5 \pm 7.5$ & $155.65 \pm 6.29$ & $12.8 \pm 11.2$ & $3.76 \pm 0.71$ \\
\hline Hondo & HWW & $53.3 \pm 26.2$ & $66.25 \pm 47.24$ & $13.0 \pm 12.5$ & $2.97 \pm 0.83$ \\
\hline Winterhawk & HWW & $31.7 \pm 10.3$ & $10.11 \pm 4.59$ & $13.3 \pm 6.3$ & $6.63 \pm 0.54$ \\
\hline Arapahoe & HWW & $38.3 \pm 10.3$ & $39.81 \pm 16.63$ & $13.3 \pm 6.3$ & $5.96 \pm 2.40$ \\
\hline SD07W053 & HWW & $69.0 \pm 34.7$ & $169.81 \pm 113.89$ & $13.3 \pm 9.4$ & $5.66 \pm 0.69$ \\
\hline Hawken & HWW & $88.0 \pm 8.0$ & $62.56 \pm 19.85$ & $13.3 \pm 7.2$ & $3.72 \pm 1.80$ \\
\hline KS030024-K-4 & HWW & $97.7 \pm 1.9$ & $274.91 \pm 31.89$ & $13.3 \pm 4.7$ & $4.93 \pm 1.72$ \\
\hline Roane & SWW & - & - & $13.3 \pm 5.5$ & $2.42 \pm 1.80$ \\
\hline Ike & HWW & $83.3 \pm 10.3$ & $96.49 \pm 23.82$ & $14.5 \pm 9.9$ & $4.74 \pm 1.91$ \\
\hline ART & HWW & $60.0 \pm 21.6$ & $67.26 \pm 79.52$ & $14.5 \pm 14.9$ & $4.74 \pm 1.62$ \\
\hline Santa Fe & HWW & $93.3 \pm 2.4$ & $143.88 \pm 53.13$ & $14.5 \pm 5.5$ & $5.47 \pm 2.19$ \\
\hline P04287A1-10 & SWW & - & - & $15.0 \pm 7.1$ & $3.79 \pm 1.47$ \\
\hline NW05M6015-25-4 & HWW & $81.7 \pm 22.5$ & $122.02 \pm 86.03$ & $15.0 \pm 14.6$ & $5.22 \pm 3.81$ \\
\hline INW0411 ${ }^{\mathrm{d}}$ & SWW & - & - & $30.0 \pm 18.9$ & $1.93 \pm 0.52$ \\
\hline P02444A1-23-9d & SWW & - & - & $30.0 \pm 17.3$ & $1.99 \pm 0.48$ \\
\hline
\end{tabular}

a Values represent mean trait value and its standard error for each lines; - indicates missing data.

${ }^{\mathrm{b}} \mathrm{HWW}=$ hard winter wheat and SWW = soft winter wheat.

c Accessions with DON at $<5 \mathrm{mg} / \mathrm{kg}$ in greenhouse experiments.

${ }^{\mathrm{d}}$ Accessions with DON at $<2 \mathrm{mg} / \mathrm{kg}$ in field experiments. 
A much lower DON concentration $(<30.0 \mathrm{mg} / \mathrm{kg})$ was detected from field samples than from greenhouse samples (Supplemental Table 1). Among all tested accessions in the field experiments, only $2.5 \%$ had a DON concentration $<2.0 \mathrm{mg} / \mathrm{kg}$, of which seven carried Fhbl and two (MD01W233-06-1 and IL02-18228) did not (Table 1). A total of $26.2 \%$ of the screened accessions had DON concentrations of 2.01 to $5.0 \mathrm{mg} / \mathrm{kg}$, including $17.7 \% \mathrm{HWW}$ and $8.5 \%$ SWW; thus, most U.S. winter wheat breeding lines and cultivars $(71.1 \%$ of tested accessions) had DON at $>5.0 \mathrm{mg} / \mathrm{kg}$ in the field-irrigated FHB nursery.

Relationship between FDK and DON in the greenhouse and field experiments. In the greenhouse experiments, 19 accessions with FDK $<25 \%$ also had low DON concentration $(<25.0 \mathrm{mg} / \mathrm{kg})$, in which six accessions had both reduced FDK $(<15 \%)$ and reduced DON concentration $(<5.0 \mathrm{mg} / \mathrm{kg}$ ) (Table 1$)$. In addition, 23 of the 29 accessions that had FDK of 25 to $50 \%$ also had relatively low DON concentrations $(<50.0 \mathrm{mg} / \mathrm{kg})$. High correlation coefficients were observed between FDK and DON con-
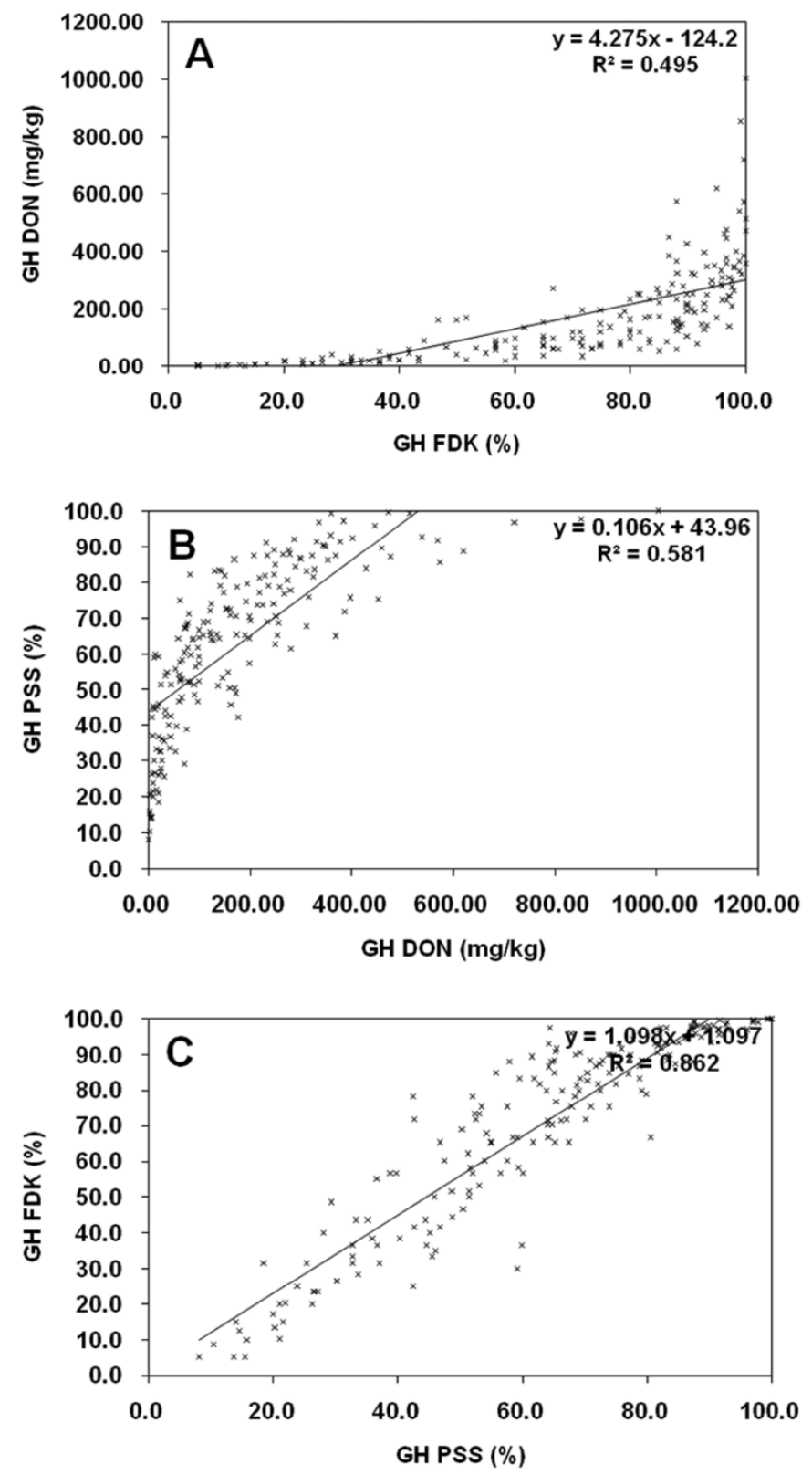

Fig. 1. Correlation coefficients of 191 accessions tested in the greenhouse (GH) experiments. A, Visually estimated Fusarium-damaged kernels (FDK) and deoxynivalenol (DON); B, DON and percentage of symptomatic spikelets (PSS) (9); and C, PSS (9) and visually estimated FDK. centration $(r=0.704, P<0.001)$ (Fig. 1A), between PSS and DON concentration $(r=0.762, P<0.001)$ (Fig. 1B), and between PSS and FDK $(r=0.928, P<0.001)$ (Fig. 1C), suggesting that wheat accessions with low PSS and low FDK usually have a low DON concentration in greenhouse experiments.

In the field experiments, correlation was also significant between mean FDK and DON concentration $(r=0.628, P<0.001)$ (Fig. 2A). Although it was lower than that from the greenhouse experiments, it was greater than the correlation between PSS and DON concentration $(r=0.503, P<0.001)$ (Fig. 2B) and between PSS and FDK $(r=0.58, P<0.001)$ (Fig. 2C) from the same experiments. The results suggest that FDK is a more accurate estimate for DON concentration than PSS in field experiments.

Contribution of Fhb1 to different FHB measurements. Four parental cultivars used in the Fhbl backcross project showed different levels of FHB infection, including Wesley (moderately susceptible in the both greenhouse and field), Trego and Clark
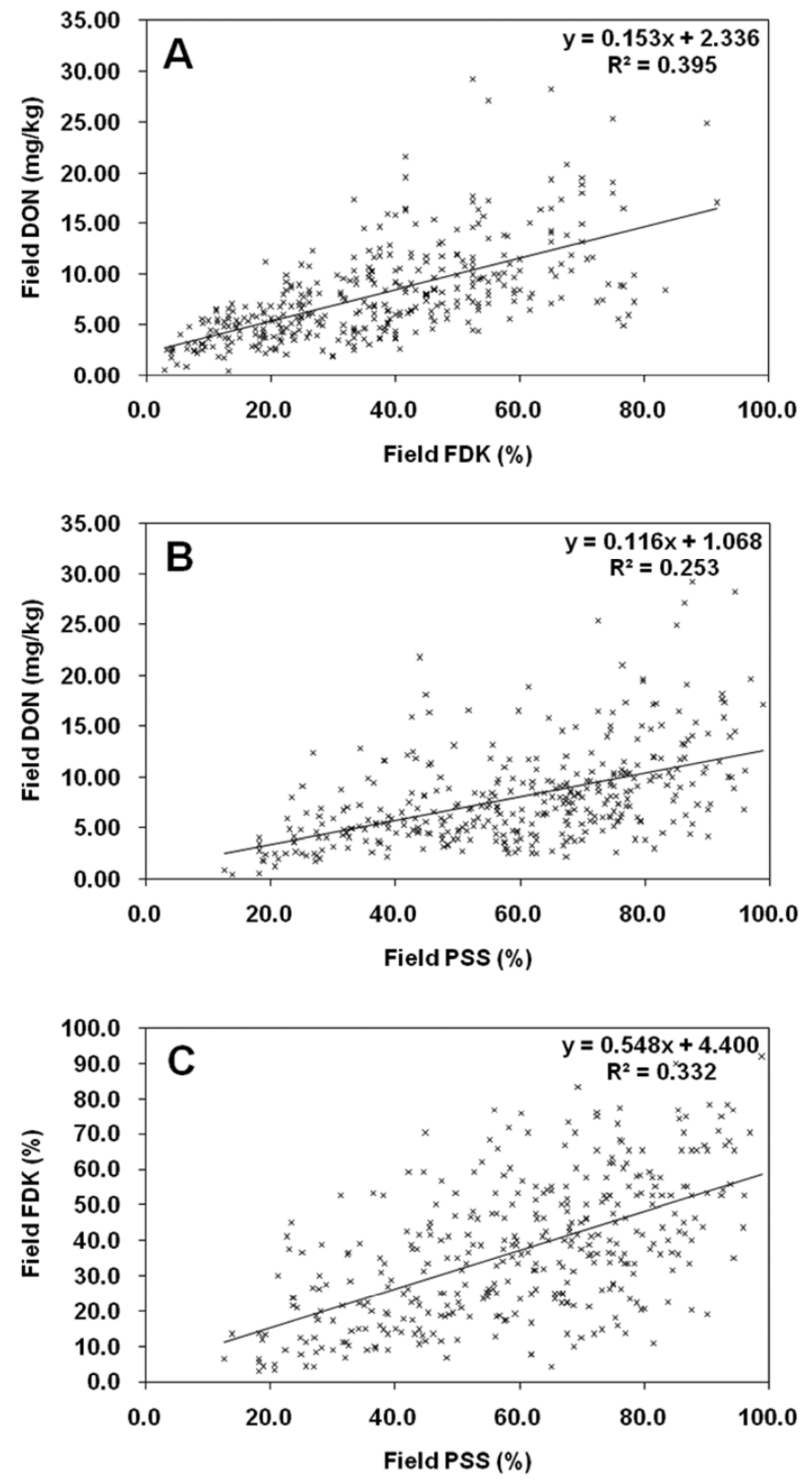

Fig. 2. Correlation coefficients of 363 wheat accessions tested in field experiments. A, Visually estimated Fusarium-damaged kernels (FDK) and deoxynivalenol (DON); B, percentage of symptomatic spikelets (PSS) (9) and DON; and C, PSS (9) and visually estimated FDK. 
(highly susceptible in the greenhouse and field), and Harding (moderately susceptible in the greenhouses but moderately resistant in the field) (Table 2). All WesleyFhb1 resistant NILs had significantly lower DON concentrations and FDK than their recurrent parent. Similar results were obtained for ClarkFhb1 and TregoFhb1 resistant NILs (Table 2). HardingFhb1 NILs showed the lowest reduction in both DON and FDK but still showed significant reductions compared with Harding; therefore, Fhbl significantly reduces both FDK and DON concentration in U.S. winter wheat genetic backgrounds.

SKNIR for FDK and DON prediction. In total, 291 wheat accessions were evaluated for FDK via SKNIR in 2011, including 100 accessions selected from set I harvested in the 2010 field experiment and 191 from set II harvested in the 2011 field experiment. The mean FDK evaluated via SKNIR was 0.8 to $86.8 \%$, similar to visual FDK estimates of 3.0 to $91.7 \%$, and the

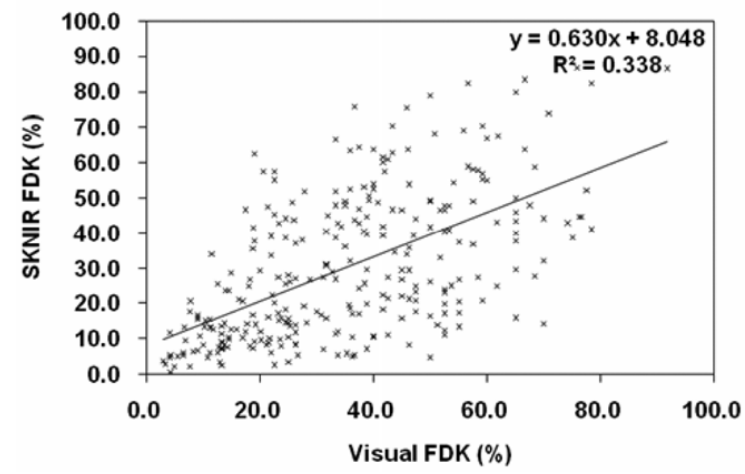

Fig. 3. Correlation between two Fusarium-damaged kernels (FDK) estimation methods: visually estimated FDK and single-kernel near-infrared (SKNIR)measured FDK in field experiments. correlation between the two methods was significant $(r=0.58$, $P<0.001$ ) (Fig. 3).

A subset of 148 wheat accessions from the samples used for SKNIR FDK analysis was selected for DON estimation, including 27 accessions from set I and 121 accessions from set II. The mean DON concentration estimated by SKNIR was 0.36 to $70.18 \mathrm{mg} / \mathrm{kg}$, with $6.8 \%$ of the accessions having $>30.0 \mathrm{mg} / \mathrm{kg}$, whereas the GC-MS method provided a narrower range of DON concentrations for the same set of samples $(0.49$ to $29.25 \mathrm{mg} / \mathrm{kg})$. The correlation coefficient between the two methods was significant $(r=0.46, P<0.001)$ (Table 3). Meanwhile, significant correlations were also observed among visual FDK, GC-MS-measured DON concentration, SKNIR-measured FDK, and SKNIR-measured DON concentration (Table 3).

\section{DISCUSSION}

A previous study evaluated PSS in both greenhouse and field experiments (9), whereas the same accessions were analyzed for FDK and DON concentration in the current study. In the green-

TABLE 3. Correlation coefficients among gas chromatography-mass spectroscopy (GC-MS)- and single-kernel near-infrared (SKNIR)-measured deoxynivalenol (DON) content and visually estimated Fusarium-damaged kernel (FDK) and SKNIR-measured FDK for 148 selected wheat accessions from both set I and set II field experiments ${ }^{\mathrm{a}}$

\begin{tabular}{lcccc}
\hline Traits & PSS & $\begin{array}{c}\text { FDK } \\
\text { (visual) }\end{array}$ & $\begin{array}{c}\text { DON } \\
\text { (GC-MS) }\end{array}$ & $\begin{array}{c}\text { FDK } \\
\text { (SKNIR) }\end{array}$ \\
\hline FDK (visual) & $0.62 * * *$ & $\ldots$ & $\ldots$ & $\ldots$ \\
DON (GC-MS) & $0.57 * * *$ & $0.74 * * *$ & $\ldots$ & $\ldots$ \\
FDK (SKNIR) & $0.38^{* * *}$ & $0.72 * * *$ & $0.49 * * *$ & $\ldots$ \\
DON (SKNIR) & $0.36^{* * *}$ & $0.68^{* * *}$ & $0.46^{* * *}$ & $0.95 * * *$ \\
\hline
\end{tabular}

a PSS = percentage of symptomatic spikelets and $* * * *$ indicates significant at the 0.001 probability level.

TABLE 2. Contribution of Fhb1 allele to low Fusarium-damaged kernel (FDK) and deoxynivalenol (DON) content in Fhb1 near-isogenic lines (NILs)

\begin{tabular}{|c|c|c|c|c|c|}
\hline \multirow[b]{2}{*}{ Accession $^{\mathrm{a}}$} & \multirow[b]{2}{*}{ Class } & \multicolumn{2}{|c|}{$\operatorname{FDK}(\%)$} & \multicolumn{2}{|c|}{$\mathrm{DON}(\mathrm{mg} / \mathrm{kg})$} \\
\hline & & Greenhouse & Field & Greenhouse & Field \\
\hline \multicolumn{6}{|l|}{ Clark } \\
\hline ClarkFhb1NIL09F-4 & SWW & 60.0 & 5.0 & 33.69 & 1.16 \\
\hline ClarkFhb1NIL09F-23 & SWW & 23.3 & 6.5 & 24.22 & 0.88 \\
\hline ClarkFhb1NIL09F-45 & SWW & 26.7 & 3.0 & 26.20 & 0.59 \\
\hline ClarkFhb1NIL-75 & SWW & 23.3 & 13.3 & 14.55 & 0.49 \\
\hline NIL mean & $\ldots$ & 33.3 & 7.0 & 24.67 & 0.78 \\
\hline Clark & SWW & 86.1 & 33.3 & 193.00 & 2.54 \\
\hline Reduction (\%) & $\ldots$ & 61.3 & 79.0 & 87.2 & 69.3 \\
\hline \multicolumn{6}{|l|}{ Trego } \\
\hline TregoFhb1NIL09S-100 & HWW & 33.3 & 4.1 & 8.58 & 1.82 \\
\hline TregoFhb1NIL09S-99 & HWW & 15.0 & 5.5 & 9.57 & 4.07 \\
\hline TregoFhb1NIL09S-98 & HWW & 10.3 & 11.3 & 5.15 & 6.46 \\
\hline Nil mean & $\ldots$ & 19.5 & 7.0 & 7.77 & 4.12 \\
\hline Trego & HWW & 88.0 & 37.5 & 234.20 & 14.70 \\
\hline Reduction (\%) & $\ldots$ & 77.8 & 81.3 & 96.7 & 72.0 \\
\hline \multicolumn{6}{|l|}{ Wesley } \\
\hline Wesley FHB1 & HWW & 15.0 & 6.5 & 6.93 & 2.7 \\
\hline WesleyFhb1NIL09S-103 & HWW & 5.3 & 9.0 & 0.42 & 2.53 \\
\hline WesleyFhb1NIL09S-104 & HWW & 8.7 & 23.8 & 2.19 & 4.23 \\
\hline WesleyFhb1NIL09S-105 & HWW & 10.0 & 4.1 & 2.22 & 2.3 \\
\hline Nil mean & $\ldots$ & 9.8 & 10.9 & 2.94 & 2.94 \\
\hline Wesley & HWW & 50.0 & 27.8 & 44.67 & 8.14 \\
\hline Reduction (\%) & $\ldots$ & 80.4 & 60.8 & 93.4 & 63.9 \\
\hline \multicolumn{6}{|l|}{ Harding } \\
\hline HardingFhb1NIL09S-109 & HWW & 30.0 & 10.0 & 11.28 & 5.48 \\
\hline HardingFhb1NIL09S-108 & HWW & 70.0 & 6.9 & 99.04 & 4.87 \\
\hline HardingFhb1NIL09S-107 & HWW & 36.7 & 19.0 & 14.67 & 6.18 \\
\hline Mean & $\ldots$ & 45.6 & 12.0 & 41.66 & 5.51 \\
\hline Harding & HWW & 78.3 & 17.8 & 82.41 & 5.87 \\
\hline Reduction (\%) & $\ldots$ & 41.8 & 32.6 & 49.4 & 6.1 \\
\hline
\end{tabular}

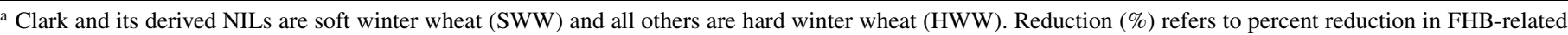
trait values due to Fhbl and is calculated by the following formula: $100 \times$ [(recurrent parent value - mean value of all its NIL)/recurrent parent value]. 
house experiments, a very high correlation was obtained between PSS and FDK (Fig. 1C), indicating high consistency between both estimates of FHB damage, which agrees with a previous report (21). High correlations detected between DON concentration and PSS and between DON and FDK (Fig. 1) supported several previous reports $(3,10,19,25)$. Visual PSS rating is done directly on growing plants; therefore, it is quicker and easier than FDK and DON estimation that needs to be done on harvested grain; thus, PSS may be a more useful estimate of FHB damage than FDK and DON measured by GC-MS in greenhouse experiments.

In contrast with the greenhouse experiments, a lower but significant correlation was detected between PSS and DON (Fig. 2B) in the field experiments. In some previous studies, significant correlation was not found between visual FHB rating and DON concentration in the field experiments $(12,26)$. The poor correlation might be because some tombstones were blown away during threshing. Threshing procedures that easily remove heavily infected grain can reduce DON concentration by reducing the proportion of DON-contaminated FDK. This is supported by a higher correlation $(r=0.628)$ between visual FDK and DON concentration than that $(r=0.503)$ between PSS and DON concentration (Fig. 2) in this study, where the thresher might have blown away some shriveled and very light grain. In addition, visual FDK was evaluated by comparing samples with known standards; therefore, FDK evaluation was more robust than the PSS evaluation in the field. In addition, field PSS scoring was based on PSS estimation of a whole plot at a specific day, not on individual plants, and personal experience can significantly affect PSS scores (21). However, threshing carefully and using a skilled person to score the PSS can improve the level of consistency between PSS and DON concentration. Visual FDK appears to be a better estimate of DON concentration than PSS in the field $(12,19,25)$.

Standard DON testing (GC-MS) is expensive and destructive, which renders it unsuitable for quick screening of a large number of materials in breeding programs; clearly, an inexpensive and effective method is needed for quick DON estimation by grain quality inspectors and wheat breeders. SKNIR spectroscopy recently has been proposed for such an application (21). A significant correlation was established between the DON concentrations that were estimated by GC-MS and SKNIR spectroscopy $(0.46$, $P<0.001)$ in the current study; however, the correlation was much lower than those $(r>0.849)$ from a previous report $(21)$. It was also lower than those reported between GC-MS and other methods such as high-performance liquid chromatography (3) and enzyme immunoassay (16). GC-MS measured DON concentration in a sample of bulked kernels, whereas the SKNIR method estimated DON concentration in a single kernel; thus, estimating DON concentration taking sample weight in consideration may improve accuracy of the SKNIR method (20). Therefore, an improved SKNIR method can be a quick and nondestructive alternative for grain quality inspectors to roughly estimate DON concentration in wheat samples and for breeders to remove DONcontaminated FDK in segregating populations.

The highest correlation was observed between SKNIRestimated FDK and DON (Table 3); thus, SKNIR FDK can be used directly to predict SKNIR DON concentration. High correlations between visual and SKNIR-estimated FDK and between visual FDK and SKNIR-estimated DON (Table 3) suggest that both visual and SKNIR FDK scores can be used to assess DON concentration in wheat samples. In addition, PSS had better correlation with visual FDK than with SKNIR FDK (Table 3); therefore, visual FDK or PSS can be used to estimate DON in most breeding programs that have no access to a SKNIR system.

In most U.S. wheat breeding programs, although Sumai 3 or its derivatives have been used as resistant parents for approximately two decades, this study shows that the majority of U.S. winter wheat, especially HWW, does not carry Fhbl and remains susceptible to FHB. Due to increased severity and frequency of FHB infection in HWW in the central Great Plains (18), transferring resistant genes from Sumai 3 and others is an effective method to quickly improve FHB resistance in U.S. HWW. In a case study, we used marker-assisted backcross to transfer Fhbl into four U.S. winter wheat cultivars and found that most selected Fhbl NILs showed significant improvement in FHB resistance. Results from the current study showed that Fhbl NILs had significantly lower FDK and DON than their recurrent parents in both the greenhouse and the field (Table 2), indicating that Fhbl can significantly reduce both FDK and DON in these genetic backgrounds. Lemmens et al. (11) reported that Fhbl detoxified DON by converting DON into nontoxic DON-3-O-glucoside and proposed detoxification as a major FHB resistance mechanism of Fhbl through either encoding a DON-glucosyltransferase or regulating enzyme expression. In another study, differential expression of UDP-glucosyltransferases between two NILs supported the hypothesis but DON concentration was not different between the NILs (8); thus, Fhbl detoxification as a major FHB resistance mechanism remains to be validated.

It is possible that the difference in DON concentration among genotypes contrasting in Fhbl alleles is due to a difference in DON production rather than detoxification. In this study, a high correlation coefficient was observed between FDK and DON concentration. A much higher proportion of FDK in harvested grain of parents than their Fhbl NILs suggests that a greater amount of fungal biomass in the parents may be responsible for a higher level of DON production than their NILs; however, this was not measured in this study.

Effect of Fhbl on reducing FDK and DON was slightly different in HardingFhb1 NILs. Although an obvious reduction was observed for both FDK and DON in both the greenhouse and field conditions, the degree of reduction was much lower $(\leq 50.0 \%)$ than in the other three sets of NILs (Table 2). The same trend was noticed for visual FHB rating (9). Thus, selecting the right recurrent parents is important for successful use of $F h b l$ in breeding for FHB resistance.

In this study, $10 \mathrm{HWW}$ accessions that do not carry Fhbl were also determined to have low FDK levels $(\leq 25.0 \%)$ and DON concentrations $(<20.13 \mathrm{mg} / \mathrm{kg})$ in the greenhouse experiments. Among them, T154 showed the lowest FDK (5.3\%) and DON concentration $(1.68 \mathrm{mg} / \mathrm{kg})$, followed by Everest. SD05085, T153, Overland, Lyman, and SD08198 also had DON concentration $<10.0 \mathrm{mg} / \mathrm{kg}$ and low FDK (Table 1). These accessions with reduced FDK and DON concentration are important native sources of resistance and the QTL underlining the resistance should be investigated further. Also, they are either newly released cultivars such as Everest and Overland or elite breeding lines that have good adaptation to U.S. HWW growing environments; thus, they are good sources of resistance to be used in FHB resistance breeding. In addition, most non- $F h b 1$ accessions from the United States that had low FDK $(<50 \%)$ and DON concentration $(<50.0 \mathrm{mg} / \mathrm{kg})$ in the greenhouse or had low FDK $(<15.0 \%)$ and DON concentration $(<5.0 \mathrm{mg} / \mathrm{kg})$ in the field experiments (Table 1) are well adapted for local wheat production; therefore, they may also contain some minor resistance QTLs and can be good parents for combining Fhbl and other Asian sources of resistance QTLs to enhance FHB resistance in U.S. HWW.

\section{ACKNOWLEDGMENTS}

This project was partly funded by the U.S. Wheat and Barley Scab Initiative and the National Research Initiative Competitive Grants CAP project 2011-68002-30029 from the USDA National Institute of Food and Agriculture. The United States Department of Agriculture (USDA) is an equal opportunity provider and employer. Mention of trade names or 
commercial products in this article is solely for the purpose of providing specific information and does not imply recommendation or endorsement by the USDA. Contribution number 13-403-J from the Kansas Agricultural Experiment Station.

\section{LITERATURE CITED}

1. Anderson, J. A., Stack, R. W., Liu, S., Waldron, B. L., Fjeld, A. D., Coyne, C., Moreno-Sevilla, B., Mitchell Fetch, J., Song, Q. J., Cregan, P. B., and Frohberg, R. C. 2001. DNA markers for Fusarium head blight resistance QTLs in two wheat populations. Theor. Appl. Genet. 102:11641168.

2. Bai, G. H., Kolb, F. L., Shaner, G. E., and Domier, L. L. 1999. Amplified fragment length polymorphism markers linked to a major quantitative trait locus controlling scab resistance in wheat. Phytopathology 89:343-348.

3. Bai, G. H., Plattner, R., Desjardins, A., and Kolb, F. 2001. Resistance to Fusarium head blight and deoxynivalenol accumulation in wheat. Plant Breed. 120:1-6.

4. Bai, G. H., and Shaner, G. E. 2004. Management and resistance in wheat and barley to Fusarium head blight. Annu. Rev. Phytopathol. 42:135-161.

5. Buerstmayr, H., Lemmens, M., Hartl, L., Doldi, L., Steiner, B., Stierschneider, M., and Ruckenbauer, P. 2002. Molecular mapping of QTLs for Fusarium head blight resistance in spring wheat. I. Resistance to fungal spread (type II resistance). Theor. Appl. Genet. 104:84-91.

6. Desjardins, A. E., Proctor, R. H., Bai, G. H., McCormick, S. P., Shaner, G., Buechley, G., and Hohn, T. M. 1996. Reduced virulence of trichothecene-nonproducing mutants of Gibberella zeae in wheat field tests. Mol. Plant-Microbe Interact. 9:775-781.

7. Fuentes, R. G, Mickelson, H. R., Busch, R. H., Dill-Macky, R., Evans, C. K., Thompson, W. G., Wiersma, J. V., Xie, W., Dong, Y., and Anderson, J. A. 2005. Resource allocation and cultivar stability in breeding for Fusarium head blight resistance in spring wheat. Crop Sci. 45:1965-1972.

8. Jia, H. Y., Cho, S., and Muehlbauer, G. J. 2009. Transcriptome analysis of a wheat near-isogenic line pair carrying Fusarium head blight-resistant and susceptible alleles. Mol. Plant-Microbe Interact. 22:1366-1378.

9. Jin, F. Zhang, D. D., Bochus, W., Baenziger, P. S., Carver, B., and Bai, G. H. 2013. Fusarium head blight resistance in U.S. winter wheat cultivars and elite breeding lines. Crop Sci. 53:2006-2013.

10. Lemmens, M., Buerstmayr, H., Krska, R., and Schuhmacher, R. 2004. The effect of inoculation treatment and long-term application of moisture on Fusarium head blight symptoms and deoxynivalenol contamination in wheat grains. Eur. J. Plant Pathol. 110:299-308.

11. Lemmens, M., Scholz, U., Berthiller, F., Dall'Asta, C., Koutnik, A., Schuhmacher, R., Adam, G., Buerstmayr, H., Mesterhazy, A., Krska, R., and Ruckenbauer, P. 2005. The ability to detoxify the mycotoxin deoxynivalenol colocalizes with a major quantitative trait locus for Fusarium head blight resistance in wheat. Mol. Plant-Microbe Interact. 18:1318-1324.

12. Liu, W. Z., Langseth, W., Skinnes, H., Elen, O. N., and Sundheim, L. 1997. Comparison of visual head blight ratings, seed infection levels, and deoxynivalenol production for assessment of resistance in cereals inoculated with Fusarium culmorum. Eur. J. Plant Pathol. 103:589-595.
13. Ma, H. X., Zhang, K. M., Gao, L., Bai, G. H., Chen, H. G., Cai, Z. X., and Lu, W. Z. 2006. Quantitative trait loci for resistance to Fusarium head blight and deoxynivalenol accumulation in Wangshuibai wheat under field conditions. Plant Pathol. 55:739-745.

14. McCartney, C. A., Somers, D. J., Fedak, G., DePauw, R. M., Thomas, J., Fox, S. J., Humphreys, D. G., Lukow, O., Savard, M. E., McCallum, B. D., Gilbert, J., and Cao, W. 2007. The evaluation of FHB resistance QTLs introgressed into elite Canadian spring wheat germplasm. Mol. Breed. 20:209-221.

15. Mesterházy, A., Bartók, T., Mirocha, C. G., and Komorócay, R. 1999. Nature of wheat resistance to Fusarium head blight and the role of deoxynivalenol for breeding. Plant Breed. 118:97-110.

16. Miedaner, T., Schneider, B., and Geiger, H. H. 2003. Deoxynivalenol (DON) content and Fusarium head blight resistance in segregating population of winter rye and winter wheat. Crop Sci. 43:519-526.

17. Mirocha, C. J., Kolaczkowski, E., Xie, W. P., Yu, H., and Jelen, H. 1998. Analysis of deoxynivalenol and its derivatives (batch and single kernel) using gas chromatography/mass spectrometry. J. Agric. Food Chem. 46:1414-1418.

18. Nopsa, J. F. H., Baenziger, P. S., Eskridge, K. M., Peiris, K. M., Kamarange, H. S., Dowell, F. E., Harris, S. D., and Wegulo, S. N. 2012. Differential accumulation of deoxynivalenol in two winter wheat cultivars varying in FHB phenotype response under field conditions. Can. J. Plant Pathol. 34:380-389.

19. Paul, P. A., Lipps, P. E., and Madden, L. V. 2005. Relationship between visual estimates of Fusarium head blight intensity and deoxynivalenol accumulation in harvested wheat grain: a meta-analysis. Phytopathology 95:1225-1236

20. Peiris, K. H. S., Dong, Y., Bockus, W. W., and Dowell, F. E. 2013. Singlekernel NIR analysis for evaluating wheat samples for Fusarium head blight resistance. Cereal Chem. Online publication. doi.org/10.1094/ CCHEM-11-12-0157-R

21. Peiris, K. H. S., Pumphrey, M. O., Dong, Y., Maghirang, E. B., Berzonsky, W., and Dowell, F. E. 2010. Near-infrared spectroscopic method for identification of Fusarium head blight damage and prediction of deoxynivalenol in single wheat kernels. Cereal Chem. 87:511-517.

22. Rudd, J. C., Horsley, R. D., McKendry, A. L., and Elias, E. M. 2001. Host plant resistance genes for Fusarium head blight: sources, mechanisms, and utility in conventional breeding systems. Crop Sci. 41:620-627.

23. Shaner, G. E. 2003. Epidemiology of Fusarium head blight of small grain cereals in North America. Pages 84-119 in: Fusarium Head Blight of Wheat and Barley 2003. American Phytopathological Society, St. Paul, MN.

24. Waldron, B. L., Moreno-Sevilla, B., Anderson, J. A., Stack, R. W., and Frohberg, R. C. 1999. RFLP mapping of QTL for Fusarium head blight resistance in wheat. Crop Sci. 39:805-811.

25. Wegulo, S. N., Bockus, W., Nopsa, J. H., De Wolf, E. D., Eskridge, K. M., Peiris, K. H. S., and Dowell, F. E. 2011. Effects of integrating cultivar resistance and fungicide application on Fusarium head blight and deoxynivalenol in winter wheat. Plant Dis. 95:554-560.

26. Wisniewska, H., Perkowski, J., and Kaczmarek, Z. 2004. Scab response and deoxynivalenol accumulation in spring wheat kernels of different geographical origins following inoculation with Fusarium culmorum. J. Phytopathol. 152:613-621. 